\title{
AVALIAÇÃO DA EXTENSÃO DA BIODEGRADAÇÃO DE PETRÓLEOS BRASILEIROS COM ÊNFASE NOS $N$-ALQUILCICLOHEXANOS
}

\author{
Laercio Lopes Martins ${ }^{\text {a,\#, Georgiana Feitosa da Cruz }}{ }^{\text {a,*, }}$, Lívia Carvalho Santos ${ }^{\mathrm{a}}$, Marcos Albieri Pudenzi ${ }^{\mathrm{b}}$ e Marcos \\ Nogueira Eberlin ${ }^{\mathrm{b}}$ \\ aLaboratório de Engenharia e Exploração de Petróleo, Centro de Ciências e Tecnologia, Universidade Estadual do Norte Fluminense, \\ 27925-535 Macaé - RJ, Brasil \\ bDepartamento de Química Orgânica, Instituto de Química, Universidade Estadual de Campinas, 13084-971 Campinas - SP, Brasil
}

Recebido em 07/10/2018; aceito em 13/12/2018; publicado na web em 24/01/2019

\begin{abstract}
ASSESSING BIODEGRADATION EXTENT OF BRAZILIAN PETROLEUM WITH EMPHASIS ON $n$-ALKYLCYCLOHEXANES. The $n$-alkylcyclohexanes are poorly evaluated in biodegradation studies of Brazilian petroleum samples. In this context, this work aimed to use the homologue series of alkylcyclohexanes to assess extend of biodegradation of 26 petroleum samples from five Brazilian sedimentary basins, analyzed by gas chromatography coupled to mass spectrometry. The $n$-alkylcyclohexanes compounds were detected in all samples, with a distribution similar to the $n$-alkane profile, in which their abundance decreasing with increasing of the biodegradation extent. Based on this, it was suggested the use of the new parameter H30/DC together with the H30/AC already proposed, calculated by the ratio between C30 hopane to the dodecyl-cyclohexane, to assess biodegradation level according Peter and Moldowan scale (PM scale). In addition, it was observed an increasing in the relative abundance of DBE 2 from class $\mathrm{O}_{2}$, analyzed by ESI(-) FT-ICR MS, mainly due to the formation of monocyclic acids with biodegradation, which correlates with the decrease of $n$-alkylcyclohexanes, a possible precursor of these acids.
\end{abstract}

Keywords: petroleum; biodegradation; $n$-alkylcyclohexanes; GC-MS; petroleomics.

\section{INTRODUÇÃO}

Os $n$-alquilciclohexanos têm sido pouco utilizados e explorados em estudos geoquímicos para avaliação de biodegradação de óleos brasileiros, embora tenham sido detectados em petróleos de diferentes bacias sedimentares brasileiras, tais como Bacia de Campos, ${ }^{1,2}$ Sergipe-Alagoas ${ }^{3}$ e Potiguar. ${ }^{4}$ A presença destes compostos em óleos e sedimentos é reconhecida há muitos anos, ${ }^{5-9}$ sendo sua série homóloga detectada em petróleos e sedimentos de bacias de todo o mundo, tais como nos extratos de rochas geradoras das Bacias McArthur, Canning, Amadeus e Hamersley da Austrália, ${ }^{10-12}$ da Bacia de Puertollano na Espanha, ${ }^{13}$ da Bacia Silesian Coal da Polônia, ${ }^{14} \mathrm{e}$ em óleos maturos dos Estados Unidos, Bolívia, Nigéria, Líbia ${ }^{8,15} \mathrm{e}$ de Alberta no Canadá. ${ }^{16}$

Os compostos $n$-alquilciclohexanos são alcanos com um anel terminal de seis carbonos, e possuem uma distribuição similar ao perfil dos $n$-alcanos. ${ }^{12,13}$ Embora esses compostos possam ter uma origem biológica direta proveniente de ácidos ciclohexilalcanóicos presentes em algumas espécies de bactérias, ${ }^{17} \mathrm{a}$ afinidade destes com os $n$-alcanos aponta para a ciclização da cadeia lateral dos lipídios como uma origem mais provável. ${ }^{8,10,11,16}$ Assim, os $n$-alquilciclohexanos têm, provavelmente, os mesmos precursores dos $n$-alcanos.

De Lima, ${ }^{2}$ ao analisar três amostras de óleos da Bacia de Campos com diferentes níveis de biodegradação (muito leve/leve, moderada e pesada), observou que a abundância relativa da série homóloga dos $n$-alquilciclohexanos tende a diminuir com o aumento da extensão da biodegradação. Essa observação está de acordo com a escala de biodegradação de Peter e Moldowan (PM), em que alquilciclohexanos têm uma alteração inicial em níveis moderados, intermediária em níveis pesados, sendo completamente degradados apenas em níveis severos de biodegradação. ${ }^{18}$

*e-mail: georgiana@lenep.uenf.br

\#e-mail alternativo: laerciolopesdm@hotmail.com
A biodegradação é um dos processos de alteração secundária que mais impactam o petróleo no interior de reservatórios em todo o mundo. ${ }^{19,20}$ Trata-se de um processo pós deposicional que ocorre em reservatório sob temperaturas de cerca de até $80{ }^{\circ} \mathrm{C}$, no qual o petróleo é biologicamente degradado em escala de tempo geológico. Os microrganismos utilizam os hidrocarbonetos e outros componentes do petróleo como fonte de carbono (doadores de elétrons) e alguns nutrientes (oxigênio molecular, nitratos, sulfatos ou íon férrico) como receptores de elétrons, ${ }^{21}$ produzindo um óleo quimicamente e fisicamente alterado e de baixa qualidade. ${ }^{22} \mathrm{~A}$ baixa qualidade do petróleo se deve, dentre outros fatores, à formação de ácidos orgânicos que ocorre devido à biodegradação dos hidrocarbonetos de forma quase sequencial e sistemática, ${ }^{22}$ iniciando-se pelos $n$-alcanos, alquilciclohexanos, isoprenóides acíclicos e compostos bicíclicos, até atingir hidrocarbonetos mais complexos com três ou mais ciclos, tais como esteranos e terpanos, em estágios mais avançados do processo. ${ }^{23}$

A presença de diversas classes de compostos numa matriz complexa como o petróleo torna necessária a utilização de técnicas analíticas capazes de separar e quantificar analitos, garantindo uma interpretação confiável das informações obtidas, principalmente quando existem diversos fatores responsáveis pela alteração nas quantidades das substâncias presentes (como origem da matéria orgânica, ambiente deposicional, fatores secundários, etc.). Nesse contexto, a cromatografia gasosa tem sido comumente utilizada para acompanhar essas mudanças composicionais, uma vez que o perfil cromatográfico ou impressão digital (fingerprint) de uma amostra de petróleo pode ser utilizado como uma das primeiras indicações qualitativas da biodegradação por meio do monitoramento da diminuição dos compostos lineares ( $n$-alcanos), levando ao aumento das razões pristano $\mathrm{Pr} / n \mathrm{C}_{17}$ e fitano $\mathrm{Fi} / n \mathrm{C}_{18}{ }^{21,24}$ Essas mudanças sistemáticas também têm sido usadas por geoquímicos do petróleo para formular escalas de biodegradação baseadas na remoção dos hidrocarbonetos de menor a maior complexidade, sendo a de Peters e Moldowan a mais amplamente utilizada, que varia de 0 (nenhuma biodegradação) 
a 10 (biodegradação severa). ${ }^{18,23}$ No entanto, classificar amostras de óleo de acordo com seu nível de biodegradação não é uma tarefa simples, devido à sua complexidade composicional, e também ao fato de que toda acumulação petrolífera é composta por mistura de óleos de diferentes maturidades, origens (diferentes rochas geradoras) ou mesmo níveis de biodegradação. ${ }^{22}$ Desta forma, a sugestão de novos parâmetros, como a utilização dos compostos $n$-alquilciclohexanos encontrados em petróleos brasileiros, pode auxiliar nesta tarefa classificatória e de importância significativa para geoquímicos..$^{2,3,4}$

Além da avaliação dos hidrocarbonetos por cromatografia gasosa, outras técnicas vêm sendo utilizadas para avaliação da biodegradação por meio da avaliação dos compostos heteroatômicos (polares), como, por exemplo, a espectrometria de massas por Orbitrap (Orbitrap FT MS $)^{25,26}$ e a espectrometria de massas de ressonância ciclotrônica de íons por transformada de Fourier (FT-ICR MS), ${ }^{27,28}$ capaz de analisar centenas de milhares de compostos do petróleo em uma única análise..$^{29}$ Dentre as muitas classes heteroatômicas avaliadas, uma das mais significantes para avaliar o processo de biodegradação é a classe $\mathrm{O}_{2}$, composta principalmente por ácidos carboxílicos. ${ }^{27}$ Os compostos polares podem ser classificados de acordo com o número de carbono ou do DBE (double bond equivalent), que corresponde ao número de insaturação (anéis mais ligações duplas). ${ }^{29}$ Por exemplo, os compostos de DBE 1 da classe $\mathrm{O}_{2}$ compreendem principalmente os ácidos lineares (uma dupla apenas referente ao grupo funcional carboxila); já os compostos de DBE 2 da classe $\mathrm{O}_{2}$ compreendem principalmente os ácidos monocíclicos (uma dupla do grupo carboxila mais um anel). ${ }^{27}$

Neste contexto, o objetivo deste trabalho foi utilizar os compostos da série homóloga dos $n$-alquilciclohexanos para avaliar a extensão da biodegradação de um conjunto de amostras de petróleos brasileiros, verificando-se a relação entre a redução na abundância desses compostos e o aumento do nível de biodegradação, com consequente formação de ácidos monocíclicos representados pelos compostos de DBE 2 presentes na classe $\mathrm{O}_{2}$.

\section{PARTE EXPERIMENTAL}

\section{Amostras}

Neste trabalho foram utilizadas 26 amostras de petróleo provenientes de seis bacias sedimentares brasileiras: Campos (C01 a C18); Santos (SA01); Solimões (SO02); Potiguar (P01); Espírito Santo (E01); e Recôncavo (R01 a R04). Essas seis bacias estão entre as principais províncias responsáveis atualmente pela produção de petróleo no Brasil. ${ }^{30}$ Essas amostras, com exceção da amostra E01, foram classificadas em níveis de biodegradação (PM) por Martins et al., ${ }^{31}$ conforme apresentado na Tabela 1.

\section{Fracionamento dos óleos}

Foi realizado o fracionamento SARA das amostras de óleo, que consiste na separação do óleo nas frações de hidrocarbonetos saturados (S) e aromáticos (A), resinas (R) e asfaltenos (A). Inicialmente, os asfaltenos foram removidos do óleo cru utilizando-se $n$-hexano como solvente para promover a precipitação, seguido por filtração, de acordo com metodologia adaptada de Martins et al..$^{32}$ Adicionaram-se 120,0 mL de $n$-hexano (grau cromatográfico, Sigma-Aldrich) a 4,0 g de óleo. A mistura foi agitada, seguindo para o banho em ultrassom por 10 minutos. Após a precipitação, a mistura malteno/asfalteno foi passada por filtro Whatman $n^{\circ} 40$. O precipitado (fração asfaltênica no filtro) foi lavado com $60,0 \mathrm{~mL}$ de $n$-hexano, com o intuito de remover os possíveis compostos adsorvidos. O solvente da fração maltênica resultante (filtrado) foi evaporado e a amostra reservada para cromatografia líquida.
Tabela 1. Código das amostras, grau API e nível de biodegradação de acordo com a escala $\mathrm{PM}^{3}$

\begin{tabular}{|c|c|c|}
\hline Amostras & API & Nível de Biodegradação \\
\hline R01 & 42 & \multirow{4}{*}{$0-1$} \\
\hline $\mathrm{R} 02$ & 39 & \\
\hline R04 & 39 & \\
\hline SO02 & 43 & \\
\hline SA01 & 30 & \multirow{2}{*}{$2-3$} \\
\hline P01 & 30 & \\
\hline $\mathrm{C} 01$ & 17 & \multirow{15}{*}{3} \\
\hline $\mathrm{C} 02$ & 24 & \\
\hline $\mathrm{C} 03$ & 24 & \\
\hline $\mathrm{C} 04$ & 21 & \\
\hline $\mathrm{C} 06$ & 26 & \\
\hline $\mathrm{C} 07$ & 20 & \\
\hline $\mathrm{C} 09$ & 24 & \\
\hline $\mathrm{C} 11$ & 20 & \\
\hline $\mathrm{C} 12$ & 21 & \\
\hline $\mathrm{C} 13$ & 26 & \\
\hline $\mathrm{C} 14$ & 26 & \\
\hline $\mathrm{C} 15$ & 24 & \\
\hline $\mathrm{C} 16$ & 23 & \\
\hline $\mathrm{C} 17$ & 22 & \\
\hline $\mathrm{C} 18$ & 24 & \\
\hline $\mathrm{C} 05$ & 20 & \multirow{3}{*}{$4-5$} \\
\hline $\mathrm{C} 08$ & 19 & \\
\hline $\mathrm{C} 10$ & 19 & \\
\hline R03 & 21 & $5-10$ \\
\hline E01 & 14 & Desconhecido \\
\hline
\end{tabular}

Parte da fração maltênica dos óleos (aproximadamente $0,10 \mathrm{~g}$ ) foi, então, submetida à cromatografia líquida em coluna de vidro recheada com sílica gel 60 (230-400 mesh; Merck) previamente ativada em estufa a $120^{\circ} \mathrm{C}$ durante $12 \mathrm{~h}$. As frações de hidrocarbonetos saturados e aromáticos e a fração contendo os compostos NSO (resinas) foram eluídas utilizando-se 40,0 $\mathrm{mL}$ de $n$-hexano, 40,0 $\mathrm{mL}$ de $n$-hexano:diclorometano ( $8: 2 \mathrm{v} / \mathrm{v})$ e 40,0 $\mathrm{mL}$ diclorometano:metanol $(9: 1 \mathrm{v} / \mathrm{v})$, respectivamente. Todos os solventes utilizados foram de grau cromatográfico, da Sigma-Aldrich (Brasil). O solvente presente nas frações coletadas foi evaporado, e as massas obtidas foram registradas.

\section{Cromatografia gasosa acoplada à espectrometria de massas (CG-EM)}

A fração dos compostos saturados foi analisada em um cromatógrafo em fase gasosa Agilent Technologies $6890 \mathrm{~N}$, equipado com uma coluna DB-5 MS (30 m x 0,25 mm x 0,25 $\mu \mathrm{m}$ de espessura do filme), acoplado a um detector seletivo de massas Agilent 5973 MSD. A programação de temperatura utilizada foi inicialmente de $60{ }^{\circ} \mathrm{C}$ por 2 minutos, seguida por um aquecimento a uma taxa de $22{ }^{\circ} \mathrm{C} \mathrm{min}^{-1}$ até $200{ }^{\circ} \mathrm{C}$, mantendo-se nessa temperatura por $3 \mathrm{mi}$ nutos, e finalmente aquecida a uma taxa de $3{ }^{\circ} \mathrm{C} \min ^{-1}$ até $300{ }^{\circ} \mathrm{C}$, com essa temperatura final mantida por 25 minutos. $\mathrm{O}$ gás $\mathrm{He}$ foi utilizado como gás carreador, com o fluxo constante de $1 \mathrm{~mL} \mathrm{~min}{ }^{-1}$. 
As temperaturas do injetor e da linha de transferência foram de 300 e $280{ }^{\circ} \mathrm{C}$, respectivamente. $\mathrm{O}$ espectrômetro de massas foi operado com energia de ionização de $70 \mathrm{eV}$ e temperatura de $230{ }^{\circ} \mathrm{C}$ para a fonte de ionização. A injeção foi realizada sem divisão de fluxo, com o volume de injeção de $1 \mu \mathrm{L}$. Utilizou-se análise em varredura linear (Scan) na faixa de 50-550 Dalton e monitoramento seletivo de íons (SIM = Single Ion Monitoring) como modo de análise para os íons fragmentos $m / z$ 83, 85, 183 e 191, para analisar as classes de $n$-alquilciclohexanos, $n$-alcanos, isoprenóides e terpanos, respectivamente. Os compostos foram identificados com base em dados da literatura, perfil cromatográfico de amostras de referência e pelos espectros de massas.

\section{Espectrometria de massas de altíssimo poder de resolução (FT-ICR MS)}

As análises foram realizadas a partir de uma solução do óleo bruto em um espectrômetro de massas de ultra-alta resolução, o LTQ FT Ultra mass spectrometer (ThermoScientific, Bremen, Germany) com um magneto de 7,2 T. Cerca de $2 \mathrm{mg}$ das amostras foram previamente dissolvidas em 1,0 mL de tolueno, e então diluídas com 1,0 mL de metanol contendo $0,2 \%$ de hidróxido de amônio. Os solventes foram obtidos da Sigma-Aldrich (St. Louis, MO), com pureza HPLC. As amostras foram inseridas no espectrômetro de massas por infusão direta no modo negativo de íons, e analisadas com uma resolução de 400.000 em uma faixa de massas de $200-1000 \mathrm{~m} / \mathrm{z}$. Foi utilizado um fluxo na seringa de $5 \mu \mathrm{L} \mathrm{min}{ }^{-1}$, a voltagem capilar de $-3,1 \mathrm{kV}$ e foram obtidos espectros pela soma de 100 transientes totalizando 6 minutos de aquisição.

Os espectros de massas obtidos no FT-ICR MS foram processados utilizando o software PetroMS. ${ }^{33}$ Esse software foi designado para calibrar e atribuir as fórmulas moleculares para séries homólogas conhecidas a partir da medida dos valores de $\mathrm{m} / \mathrm{z}$ dos compostos polares do petróleo. Para cada espectro, processamentos automatizados foram realizados para designar fórmulas moleculares aos picos com sinal/ruído maior que três $(>3)$. Os elementos permitidos foram o ${ }^{12} \mathrm{C},{ }^{13} \mathrm{C},{ }^{1} \mathrm{H},{ }^{16} \mathrm{O},{ }^{14} \mathrm{Ne}^{32} \mathrm{~S}$. O erro máximo permitido para as fórmulas moleculares foi de $1 \mathrm{ppm}$.

\section{RESULTADOS E DISCUSSÃO}

\section{Análise dos $n$-alquilciclohexanos por CG-EM}

Assim como em De Lima, ${ }^{2}$ Da Silva ${ }^{4}$ e em outros trabalhos, a série homóloga dos $n$-alquilciclohexanos foi detectada em todas as 26 amostras de petróleos (cromatograma de massas do íon $\mathrm{m} / \mathrm{z}, 83$ ), com distribuição similar ao perfil dos $n$-alcanos como mostrado na Figura 1 para amostra E01 da Bacia do Espírito Santo (os demais cromatogramas podem ser visualizados em Martins et al.,${ }^{31}$ no material suplementar). Foi identificada a série homóloga destes cicloexanos monossubstituídos do $\mathrm{C}_{13}$ ao $\mathrm{C}_{26}$, como é apresentado na Tabela 2, e um espectro de massas representativo para sua classe, referente ao decil-cicloexano, é apresentado na Figura 2.

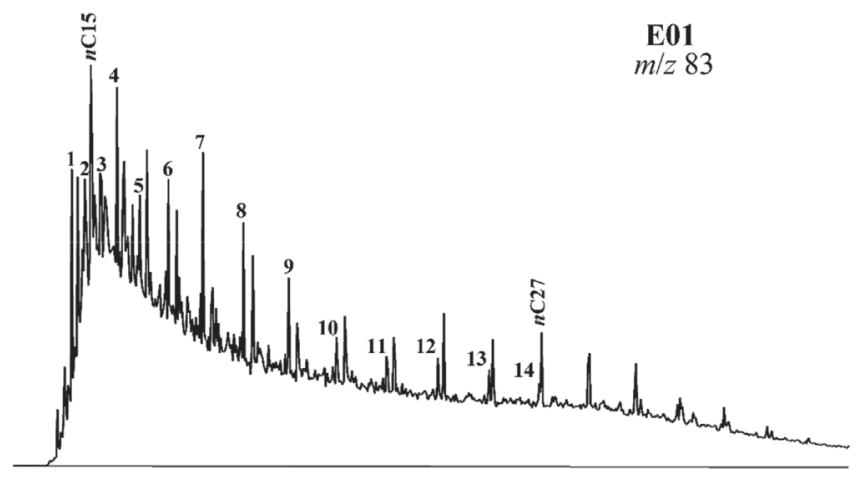

Figura 1. Cromatograma de íons reconstruídos para o íon-fragmento $\mathrm{m} / \mathrm{z}, 83$, característico dos n-alquilciclohexanos, para a amostra E01. $n C_{15}: n$-alcano $C_{15} ;{ } C_{27}: n$-alcano $C_{27}$. Os números correspondem aos compostos da série homóloga dos n-alquilciclohexanos, nomeados na Tabela 2

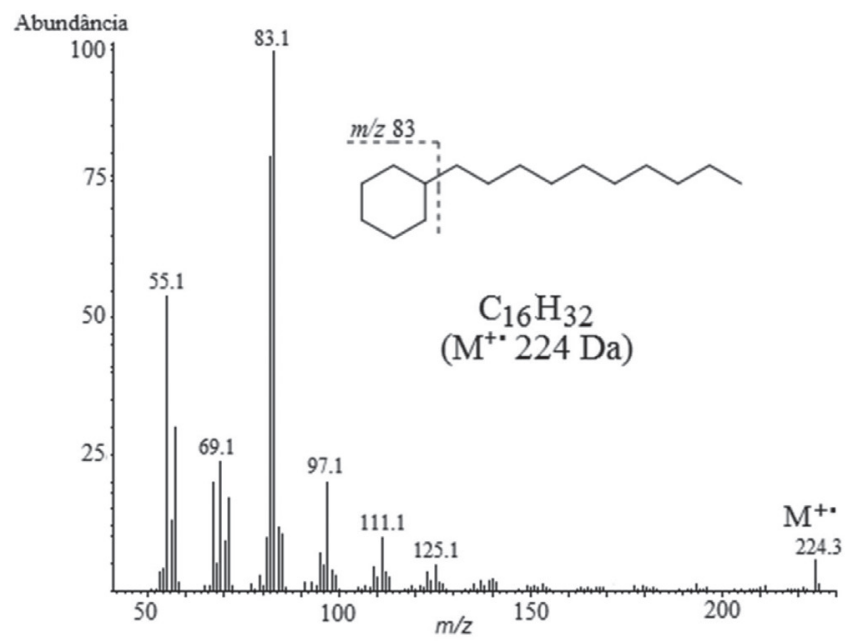

Figura 2. Espectro de massas do Decil-cicloexano (C16, pico 4), representativo da classe dos n-alquilciclohexanos

Foi observada uma nítida diminuição da abundância dos $n$-alquilciclohexanos com o aumento da extensão da biodegradação, assim como observado por De Lima, ${ }^{2}$ alcançando baixíssimas abundâncias para a amostra R03, a mais biodegradada, como já foi mostrado por Martins et al. ${ }^{31}$ (Figura 3).

Desta forma, para avaliar o nível de biodegradação utilizando esta

Tabela 2. $n$-Alquilciclohexanos detectados nas amostras de petróleo deste trabalho, monitorando-se o íon fragmento $\mathrm{m} / z$, 83

\begin{tabular}{|c|c|c|c|c|c|c|c|}
\hline Pico & Composto & Fórmula & MM & Pico & Composto & Fórmula & MM \\
\hline 1 & Heptil-cicloexano & $\mathrm{C}_{13} \mathrm{H}_{26}$ & 182 & 8 & Tetradecil-cicloexano & $\mathrm{C}_{20} \mathrm{H}_{40}$ & 280 \\
\hline 2 & Octil-cicloexano & $\mathrm{C}_{14} \mathrm{H}_{28}$ & 196 & 9 & Pentadecil-cicloexano & $\mathrm{C}_{21} \mathrm{H}_{42}$ & 294 \\
\hline 3 & Nonil-cicloexano & $\mathrm{C}_{15} \mathrm{H}_{30}$ & 210 & 10 & Hexadecil-cicloexano & $\mathrm{C}_{22} \mathrm{H}_{44}$ & 308 \\
\hline 4 & Decil-cicloexano & $\mathrm{C}_{16} \mathrm{H}_{32}$ & 224 & 11 & Heptadecil-cicloexano & $\mathrm{C}_{23} \mathrm{H}_{46}$ & 322 \\
\hline 5 & Undecil-cicloexano & $\mathrm{C}_{17} \mathrm{H}_{34}$ & 238 & 12 & Octadecil-cicloexano & $\mathrm{C}_{24} \mathrm{H}_{48}$ & 336 \\
\hline 6 & Dodecil-cicloexano & $\mathrm{C}_{18} \mathrm{H}_{36}$ & 252 & 13 & Nonadecil-cicloexano & $\mathrm{C}_{25} \mathrm{H}_{50}$ & 350 \\
\hline 7 & Tridecil-cicloexano & $\mathrm{C}_{19} \mathrm{H}_{38}$ & 266 & 14 & Eicosil-cicloexano & $\mathrm{C}_{26} \mathrm{H}_{52}$ & 364 \\
\hline
\end{tabular}

Os picos foram nomeados baseando-se em espectros de massas (Figura 2) e por comparação com a literatura. ${ }^{4}$ 


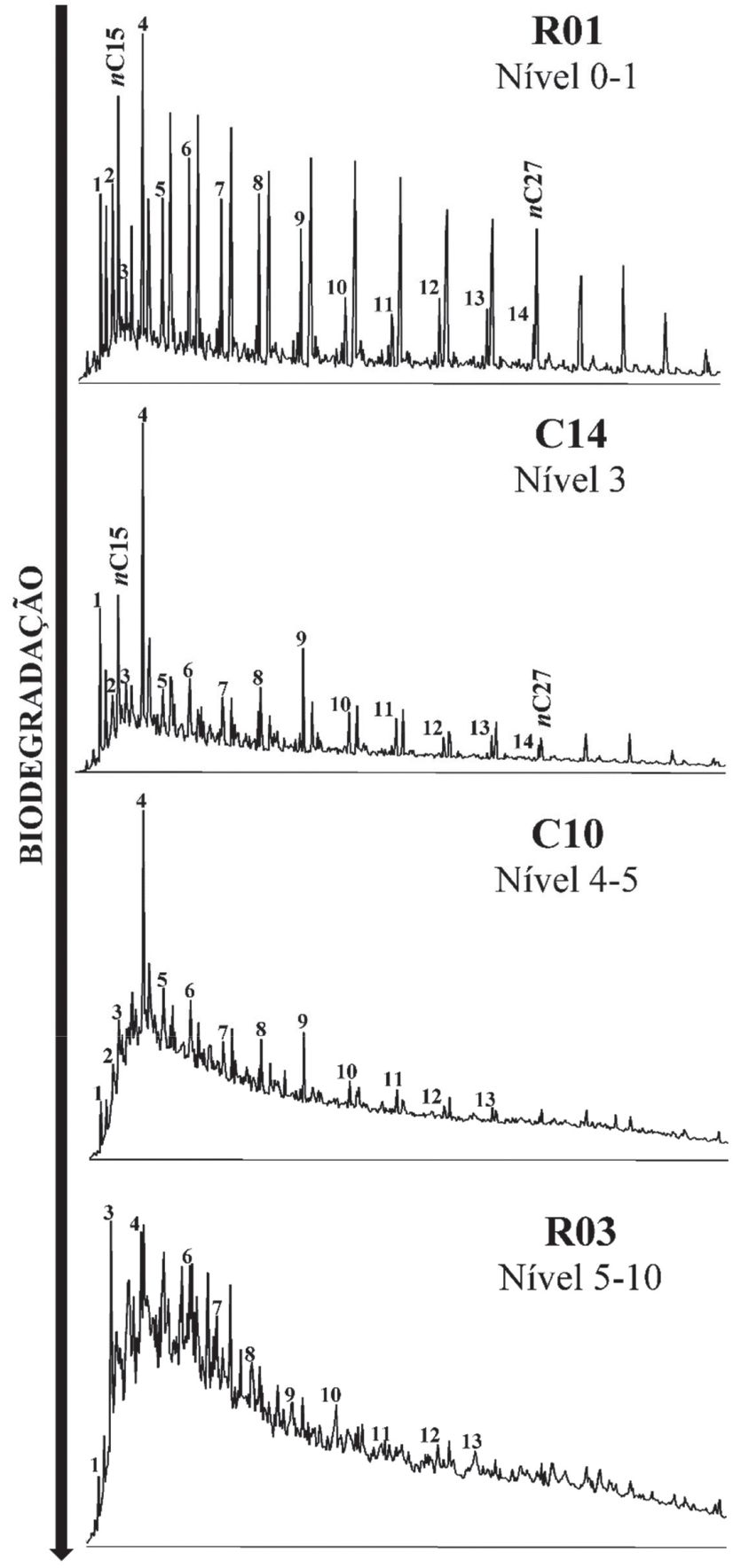

Figura 3. Cromatogramas de íons reconstruídos para o íon-fragmento $\mathrm{m} / \mathrm{z} 83$ de amostras de petróleo com diferentes níveis de biodegradação: R01, nível 0-1; C14, nível 3; C10, nível 4-5; R03, nível 5-10 (Modificado de Martins et al. ${ }^{31}$ ). $n C_{15}:$ n-alcano $C_{15} ; n C_{27}: n$-alcano $C_{27}$; Os números correspondem aos compostos da série homóloga dos n-alquilciclohexanos (Tabela 2)

classe de compostos sugere-se a utilização da razão H30/DC em conjunto com a razão H30/AC. Esses parâmetros são calculados pela razão entre a abundância do biomarcador $\mathrm{H} 3017 \alpha(\mathrm{H}), 21 \beta(\mathrm{H})$-hopano (obtido pelo cromatograma de massas do íon-fragmento $\mathrm{m} / \mathrm{z}, 191$ ) sobre o decil-cicloexano (DC) e sobre a soma da abundância dos $n$-alquilciclohexanos $\left(\mathrm{AC}=\Sigma \mathrm{C}_{13}-\mathrm{C}_{26} n\right.$-alquilciclohexanos) (obtidos pelo cromatograma de massas do íon-fragmento $\mathrm{m} / \mathrm{z} 83$ ), respectivamente. O decil-cicloexano foi escolhido para o cálculo da primeira razão, visto que se mostrou mais resistente à biodegradação. $\mathrm{O} H 30$ foi utilizado no cálculo destas razões por ser considerado um biomarcador altamente resistente à biodegradação comumente encontrado em amostras de petróleo. Na Figura 4 é apresentado um gráfico da razão $\mathrm{H} 30 / \mathrm{AC}$ versus $\mathrm{H} 30 / \mathrm{DC}$ para as 26 amostras de óleo.

Observa-se, na Figura 4, que a partir das razões propostas pôde-se classificar as amostras em quatro grupos com níveis distintos de biodegradação de acordo com a escala PM: Nível 0-2 (nenhum/muito levemente/levemente) para as amostras R01, R02, R04 e SO02, em que não houve alteração dos $n$-alquilciclohexanos; Nível 3 (moderado) para as amostras SA01, P01, E01, C01-C04, C06, C07, C09, C11-C18, em que houve alteração inicial dos $n$-alquilciclohexanos; Nível 4-5 (pesado) para as amostras C05, C08, C10, em que houve alteração intermediária destes; e Nível 6-10 (severo) para amostra R03, em que houve remoção quase completa destes compostos. Esses resultados estão em concordância com a avaliação da extensão da biodegradação realizada por Martins et al.,${ }^{31}$ com exceção da amostra P01, que havia sido classificada como levemente biodegradada. Por isso ressalta-se a importância de se avaliar várias famílias de compostos para se obter uma classificação, quanto ao nível de biodegradação, mais satisfatória. Assim, sugere-se a inclusão dessas razões para obter uma análise mais ampla e completa da extensão da biodegradação.

A fim de verificar a eficácia dos parâmetros propostos baseados nos $n$-alquilciclohexanos, esses foram relacionados com a razão do H30 $17 \alpha(\mathrm{H}), 21 \beta(\mathrm{H})$-hopano sobre o isoprenóide fitano $(\mathrm{H} 30 / \mathrm{Fi})$. Essa razão foi baseada no trabalho de Koopmans et al. ${ }^{34}$ que avaliaram a biodegradação de um conjunto de óleos pesados da Bacia Liaohe, noroeste da China, por meio da razão H30 $17 \alpha(\mathrm{H}), 21 \beta(\mathrm{H})$ hopano/(P + Fi), e já foi utilizada para corroborar a classificação do nível de biodegradação no trabalho de Martins et al. ${ }^{31}$ A Figura 5 apresenta os gráficos do parâmetro $\mathrm{H} 30 / \mathrm{Fi}$ versus os parâmetros $\mathrm{H} 30 / \mathrm{AC}$ e H30/DC.

Observa-se, na Figura 5, uma boa relação entre os parâmetros avaliados, com os valores das razões aumentando com a biodegradação devido à degradação do fitano e dos $n$-alquilciclohexanos, com exceção da amostra E01, da Bacia do Espírito Santo. Já a amostra R03 não foi mostrada nos gráficos, já que, devido ao seu nível severo de biodegradação, os valores para as razões foram discrepantes das demais, sendo de 72,40 para $\mathrm{H} 30 / \mathrm{Fi}, 0,49$ para $\mathrm{H} 30 / \mathrm{AC}$ e de 2,82 para H30/DC.

A amostra E01, que não apresentou boa correlação entre os parâmetros H30/DC e H30/Fi, é a que possui o menor grau API entre todas amostras $\left(14^{\circ}\right)$, sendo classificada como óleo asfáltico, de acordo com esse parâmetro. ${ }^{35}$ No entanto, foi classificada com nível de biodegradacão moderado (3), de acordo com os parâmetros H30/AC e H30/DC. Para verificar a possibilidade de mistura de óleos com níveis de biodegradação distintos (envolvendo petróleos leves e petróleos muito biodegradados), foi avaliada a presença do biomarcador $17 \alpha(\mathrm{H}), 21 \beta(\mathrm{H})-25$-norhopano $(25 \mathrm{NH})$ nessa amostra. A classe dos $25 \mathrm{NH}$ já havia sido detectada nas demais amostras com baixíssima abundância relativa, com valores da razão $25 \mathrm{NH} / \mathrm{H} 30$ menores que 0,08 (Martins et al.). ${ }^{31}$ No entanto, essa razão apresentou um alto valor para a amostra E01 $(25 \mathrm{NH} / \mathrm{H} 30=1,16)$. Portanto, essa amostra pode ser considerada uma mistura de óleos de diferentes níveis de biodegradação, visto que foi detectada a presença de $n$ -alcanos (verificados pelo cromatograma de massas do íon-fragmento $\mathrm{m} / \mathrm{z}, 85$ ), alcanos isoprenóides e 25 -norhopanos. ${ }^{36}$

\section{Análise dos ácidos monocíclicos por ESI(-) FT-ICR MS}

Muitos trabalhos têm indicado que a biodegradação é o principal processo que gera os ácidos carboxílicos presentes no petróleo, a partir da oxidação de seus componentes, principalmente dos hidrocarbonetos. ${ }^{19,37} \mathrm{~A}$ avaliação dos compostos $n$-alquilciclohexanos para as 26 amostras de óleo deste trabalho mostraram que a abundância 


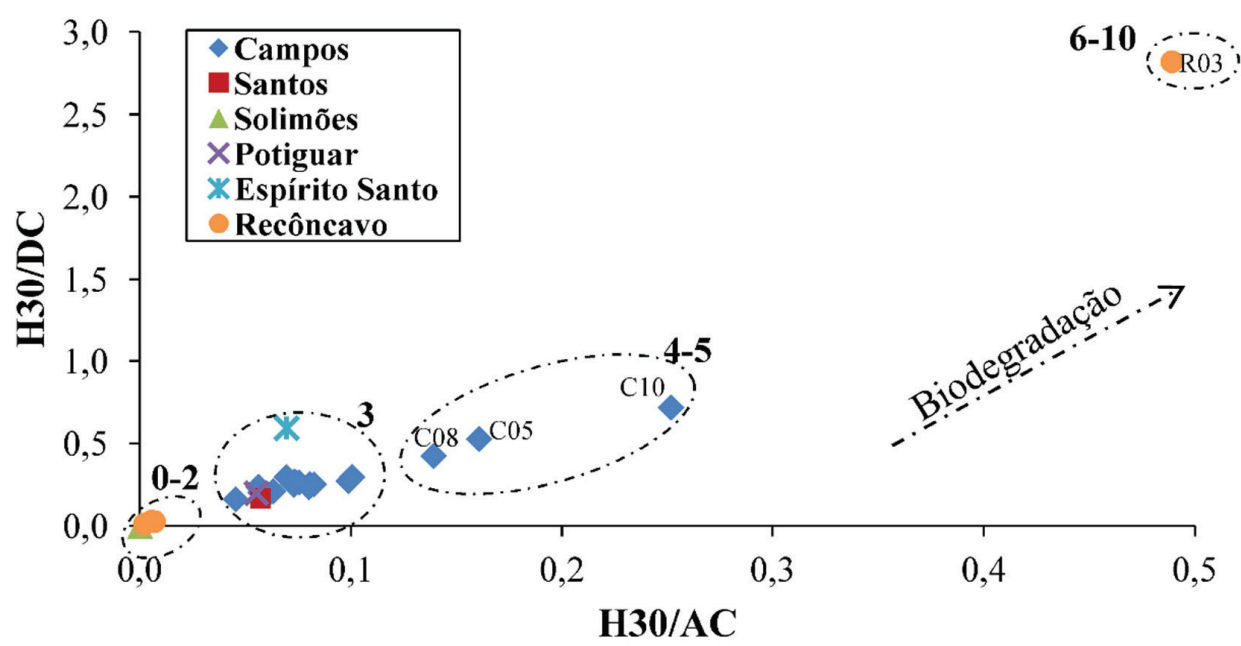

Figura 4. Gráfico da relação entre os parâmetros de biodegradação H30/AC e H30/DC, referentes às amostras de óleo brasileiro estudadas
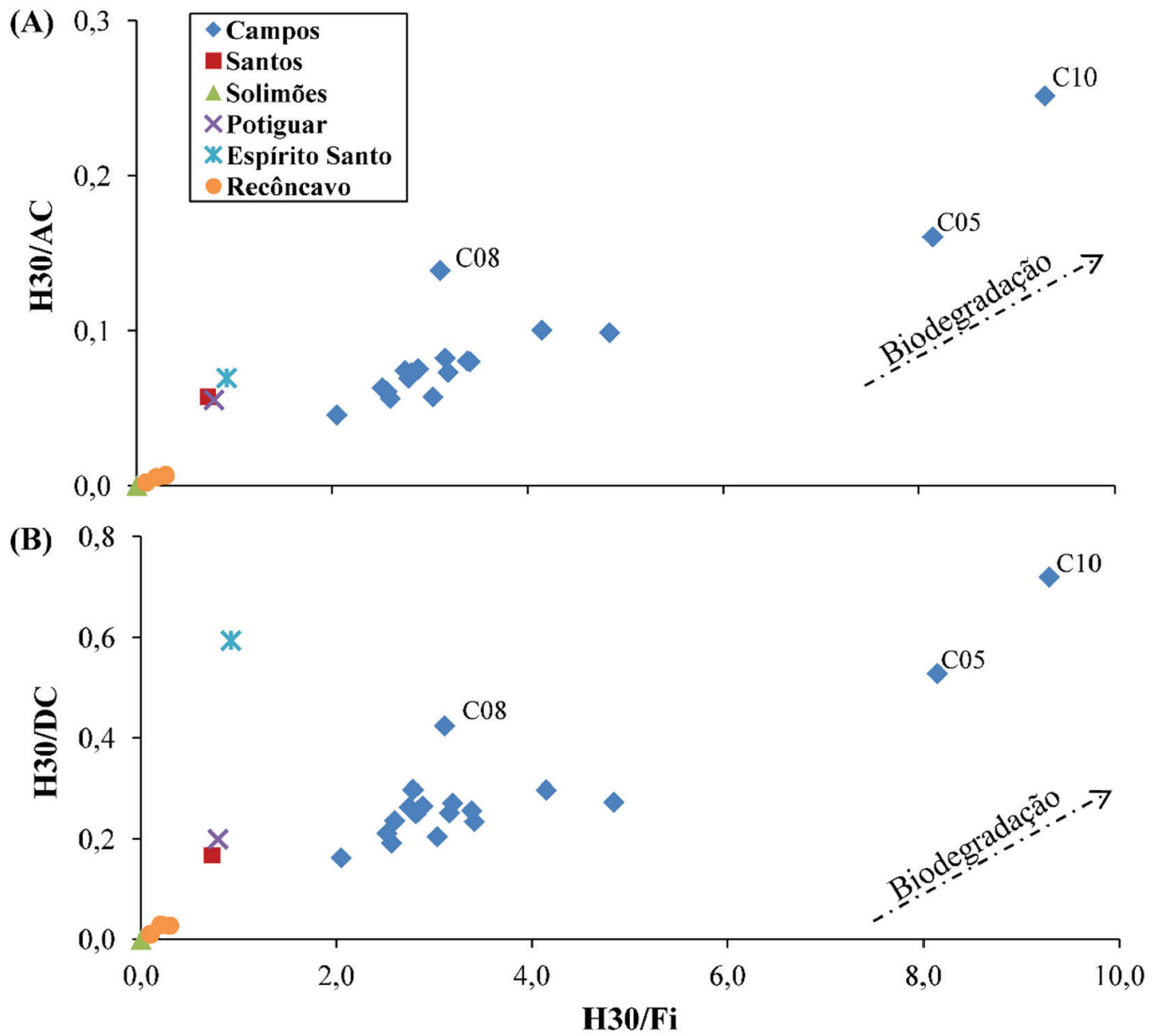

Figura 5. Gráficos da relação entre os parâmetros de biodegradação H30/AC e H30/Fi (A), e H30/DC e H30/Fi (B), referentes às amostras de óleo brasileiro estudadas

desses compostos diminui com a biodegradação e, com isso, espera-se que a abundância dos ácidos monocíclicos, os prováveis produtos desta degradação microbiana, aumente em suas composições. Para verificar essa tendência, as amostras foram analisadas por FT-ICR MS utilizando a técnica de ionização por electrospray no modo negativo, ESI(-).

Pode-se considerar que os ácidos monocíclicos presentes na classe $\mathrm{O}_{2}$ são os compostos de DBE 2, ou seja, com uma insaturação e um ciclo saturado. Desse modo, relacionou-se o parâmetro \%DBE 2 com a razão H30/AC, como mostrado no gráfico da Figura 6.

Observa-se na Figura 6A uma relação entre a abundância relativa dos compostos da classe $\mathrm{O}_{2}$ de DBE 2 com a razão H30/AC, na qual a diminuição dos compostos $n$-alquilciclohexanos indicada por essa última razão relaciona-se com o aumento dos ácidos monocíclicos. Essa tendência também foi observada por Angolini et $a l .{ }^{25}$ ao avaliarem os efeitos da biodegradação sobre compostos 

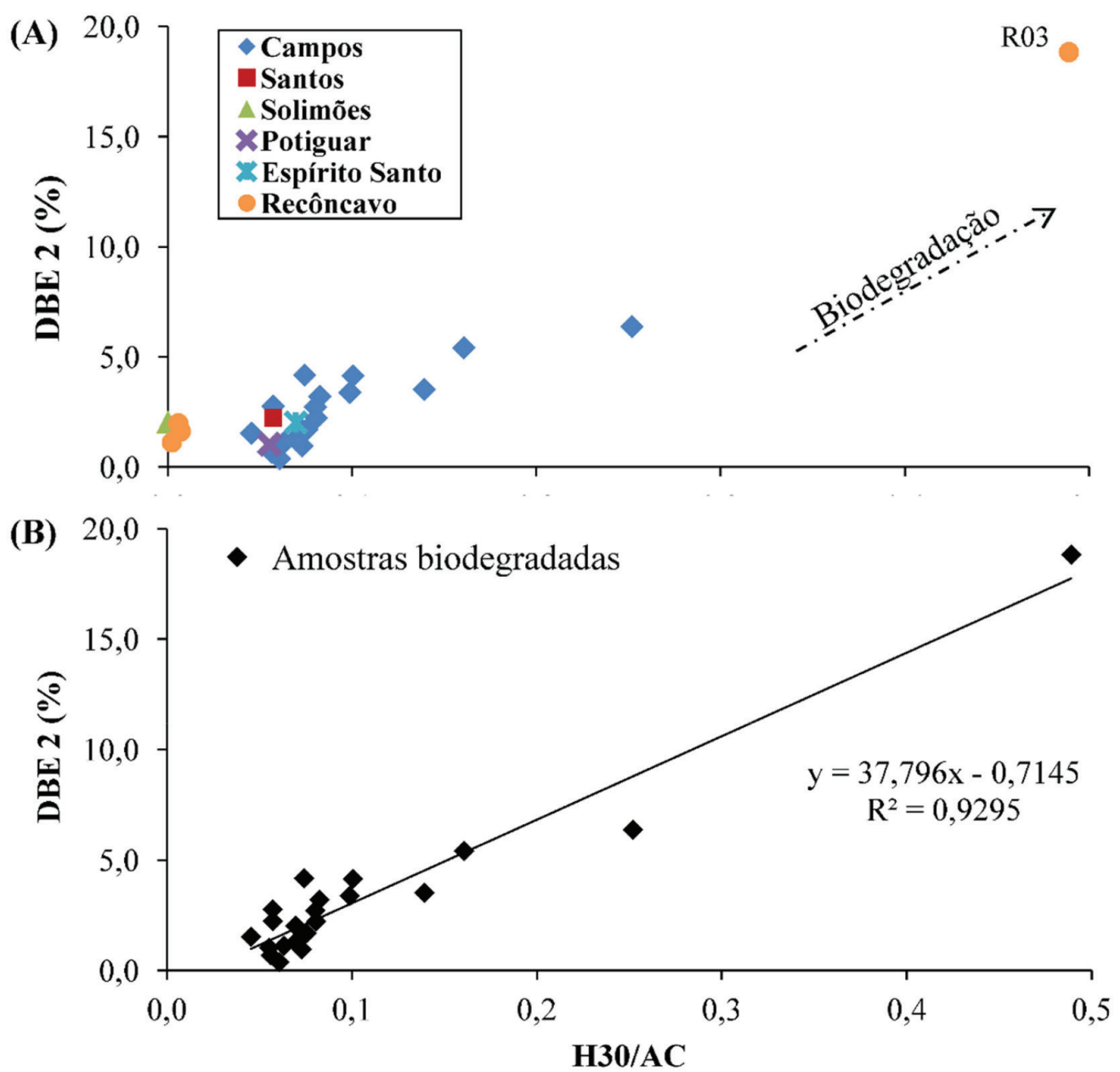

Figura 6. (A) Gráfico da abundância relativa dos compostos da classe $\mathrm{O}_{2}$ de DBE 2 (\%DBE 2) versus o parâmetro H30/AC, referentes as 26 amostras estudadas. (B) Gráfico mostrando a boa correlação entre a \%DBE 2 e o parâmetro H30/AC, para as amostras estudadas, com exceção das amostras menos biodegradadas SO01, R01, R02 e R04

oxigenados de petróleos brasileiros originados de bacias marginais utilizando Orbitrap como analisador de massas. Outra observação interessante na Figura 6A é a de que os ácidos monocíclicos foram também detectados, mesmo que em baixa abundância, nas amostras não/muito levemente biodegradadas das Bacias de Solimões e Potiguar (SO01, R01, R02, R04), que tiveram os valores para a razão H30/AC muito próximos de zero. Essa observação está coerente com o fato de que existem outras prováveis origens dos ácidos carboxílicos presentes no petróleo além da degradação microbiana, tais como os ácidos originais formados da rocha geradora (ácidos primários).$^{38} \mathrm{Já}$ na Figura 6B apresenta-se esses resultados apenas para as amostras biodegradadas, em que se pode observar uma boa correlação entre a formação dos ácidos monocíclicos e a degradação dos seus possíveis precursores, os $n$-alquilciclohexanos, mostrada pela razão H30/AC, com um modelo linear ajustado com coeficiente de determinação (ajustamento) maior que $90 \%\left(\mathrm{R}^{2}=0,93\right)$.

\section{CONCLUSÃO}

A série homóloga dos $n$-alquilciclohexanos foi detectada em todas as amostras de petróleo deste trabalho, no qual a análise desses compostos por CG-EM se mostrou útil na avaliação da extensão da biodegradação, visto que se observou a diminuição de sua abundância com o aumento da extensão deste processo microbiológico. Foi proposto o uso em conjunto de dois parâmetros para avaliar a extensão da biodegradação baseados nos $n$-alquilciclohexanos, o H30/AC e o
H30/DC, que possibilitou a classificação das amostras de petróleos brasileiros deste trabalho nos seguintes níveis de acordo com a escala de Peters e Moldowan: nível 0-2 (nenhum/muito leve/leve); nível 3 (moderado); nível 4-5 (pesado); 6-10 (severo).

A boa correlação entre o parâmetro \% DBE 2, obtido da análise dos óleos por ESI(-) FT-ICR MS, e a razão H30/AC, obtida da análise dos óleos por CG-EM, também comprovou a degradação dos $n$-alquilciclohexanos, sendo esses os prováveis precursores dos ácidos monocíclicos (compostos de DBE 2 da classe $\mathrm{O}_{2}$ ) formados devido à biodegradação.

\section{AGRADECIMENTOS}

Este trabalho teve o apoio do Laboratório de Engenharia e Exploração de Petróleo da Universidade Estadual Norte Fluminense Darcy Ribeiro (LENEP/UENF) e do Laboratório ThoMSon de Espectrometria de Massas da Universidade Estadual de Campinas (Unicamp). CENPES/Petrobras forneceram a infraestrutura necessária para conduzir a pesquisa, que foi financiada pelo PRH20-ANP, pela Capes e pela FAPERJ.

\section{REFERÊNCIAS}

1. Nascimento, L. R.; Rebouças, L. M. C.; Koike, L.; Reis, F. A. M.; Soldan, A. L.; Cerqueira, J.; Marsaioli, A. J.; Org. Geochem. 1999, 30, 1175 . 
2. De Lima, S. G.; Tese de Doutorado, Universidade Estadual de Campinas, Brasil, 2005.

3. Alves, P. B.; Tese de Doutorado, Universidade Estadual de Campinas, Brasil, 1997.

4. Da Silva, A. A.; Tese de Doutorado, Universidade Estadual de Campinas, Brasil, 2008.

5. Mair, B. J.; Eberly, P. E.; Li, K.; Rossini, F. D.; Ind. Eng. Chem. 1958 , $50,115$.

6. Hood, A.; Clerc, R. J.; O`Neal, M. J.; J. Inst. Petrol. 1959, 45, 168.

7. Johns, R. B.; Belsky, T.; McCarthy, E. D.; Burlingame, A. L.; Haug, P.; Schnoes, H. K.; Richter, W.; Calvin, M.; Geochim. Cosmochim. Acta 1966, 30, 1191.

8. Fowler, M. G.; Abolins, P.; Douglas, A. G.; Org. Geochem.1986, 10, 815 .

9. Dong, J.; Vorkink, W. P.; Lee, M. L.; Geochim. Cosmochim. Acta 1993, $57,837$.

10. Hoffmann, C. F.; Foster, C. B.; Powell, T. G.; Summons, R. E.; Geochim. Cosmochim. Acta 1987, 51, 2681.

11. Summons, R. E.; Powell, T. G.; Boreham, C. J.; Geochim. Cosmochim. Acta 1988, 52, 1747.

12. Brocks, J. J.; Buick, R.; Summons, R. E.; Logan, G. A.; Geochim. Cosmochim. Acta 2003, 22, 4321.

13. Del Rio, J. C.; Garcia-Molla, J.; Gonzalez-Vila, F. J.; Martin, F.; Org. Geochem. 1994, 21, 897.

14. Fabianska, M. J.; Bzowska, G.; Matuszewska, A.; Racka, M.; Skret, U.; Chem. Erde 2003, 63, 63.

15. Kissin, Y. V.; Org. Geochem. 1990, 15, 575.

16. Rubinstein, I.; Strausz, O. P.; Geochim. Cosmochim. Acta 1979, 43, 1387.

17. Suzuki, K. I.; Saito, K.; Kawaguchi, A.; Okuda, S.; Komagata, K.; J. Gen. Appl. Microbiol. 1981, 27, 261.

18. Peters, K. E.; Moldowan, J. M.; Org. Geochem. 1991, 17, 47.

19. Wenger, L. M.; Davis, C. L.; Isaksen, G. H.; SPE Reservoir Eval. Eng. 2002, 5,375 .

20. Larter, S.; Huang, H.; Adams, J.; Bennet, B.; Jokanola, O.; Oldenburg, T. B. P.; Jones, M.; Head, I.; Riediger, C.; Fowler, M. G.; AAPG Bull. 2006, 90, 921 .

21. Da Cruz, G. F.; Marsaioli, A. J.; Quim. Nova 2012, 35, 1628.
22. Head, I. M.; Jones, D. M.; Larter, S. R.; Nature 2003, 426, 344.

23. Head, I. M.; Larter, S. R.; Gray, N. D.; Sherry, A.; Adams, J. J.; Aitken, C. M.; Jones, D. M.; Rowan, A. K.; Huang, H.; Roling, W. F. M. Em Hydrocarbon Degradation in Petroleum Reservoirs; Timmis, K. N., eds.; Springer-Verlag: Berlin Heidelberg, 2010, cap. 54.

24. Peters, K. E.; Walters, C. C.; Moldowan, J. M.; The biomarker guide: Biomarkers and Isotopes in the Environment and Human History, $2^{\text {nd }}$ ed., Cambridge University Press: United Kingdom, 2005.

25. Angolini, C. F. F.; Capilla, R.; Marsaioli, A. J.; Energy Fuel 2015, 29, 4886.

26. Martins, L. L.; Angolini, C. F. F.; Da Cruz, G. F.; Marsaioli, A. J.; J. Braz. Chem. Soc. 2017, 28, 848.

27. Kim, S.; Stanford, L. A.; Rodgers, R. P.; Marshall, A. G.; Walters, C. C.; Qian, K.; Wenger, L. M.; Mankiewicz, P.; Org. Geochem. 2005, 36, 1117.

28. Vaz, B. G.; Silva, R. C.; Klitzke, C. F.; Simas, R. C.; Nascimento, H. D. L.; Garcia, D. F.; Eberlin, M. N.; Azevedo, D. A.; Energy Fuels 2013 , 27, 1277.

29. Marshall, A. G.; Rodgers, R. P.; Acc. Chem. Res. 2004, 37, 53.

30. Agência Nacional do Petróleo, Boletim da Produção de Petróleo e Gás Natural; Junho 2018, 70; PP 1-26.

31. Martins, L. L.; Pudenzi, M. A.; Da Cruz, G. F.; Nascimento, H. D. I.; Eberlin, M. N.; Energy Fuels 2017, 31, 6649.

32. Martins, L. L.; Franklin, G. C.; De Souza, E. S.; Da Cruz, G. F.; Quim. Nova 2014, 37, 1263.

33. Corilo, Y. E.; Vaz, B. G.; Simas, R. C.; Nascimento, H. D. L.; Klitzke, C. F.; Pereira, R. C. L.; Bastos, W. L.; Santos Neto, E. V.; Rodgers, R. P.; Eberlin, M. N.; Anal. Chem. 2010, 82, 3990.

34. Koopmans, M. P.; Larter, S. R.; Zhang, C.; Mei, B.; Wu, T.; Chen, Y.; AAPG Bull. 2002, 86, 1833.

35. Farah, M. A. Em O Petróleo; Brasil, N. I., Araújo, M. A. S., Sousa, E. C. M., eds.; LTC: Rio de Janeiro, 2014, cap. 2.

36. Bennet, B.; Fustic, M.; Farrimond, P.; Huang, H.; Larter, S. R.; Org. Geochem. 2006, 37, 787.

37. Meredith, W.; Kelland, S. J.; Jones, D. M.; Org. Geochem. 2000, 31, 1059.

38. Watson, J. S.; Jones, D. M.; Swannell, R. P. J.; Van Duin, A. C. T.; Org. Geochem. 2002, 33, 1153. 